\title{
Amplified emission of phthalocyanine isolated in cryogenic matrices
}

\author{
Nadia Dozova, ${ }^{a}$ Ciaran Murray, ${ }^{a}$ John G. McCaffrey, ${ }^{* a}$ Niloufar Shafizadeh ${ }^{b}$ and \\ Claudine Crépin $* b$
}

\author{
Received 12th December 2007, Accepted 28th January 2008 \\ First published as an Advance Article on the web 21st February 2008 \\ DOI: $10.1039 / b 718418 b$
}

Laser induced fluorescence spectroscopy of free-base $\left(\mathrm{H}_{2} \mathrm{Pc}\right)$ and zinc $(\mathrm{ZnPc})$ phthalocyanines trapped in rare gas and nitrogen matrices reveals a quite unexpected phenomenon with a moderate increase in the laser intensity. In all matrices except Xe, a huge increase occurs in the intensity of an emission band near $755 \mathrm{~nm}$ when pumping the $\mathrm{S}_{1} \leftarrow \mathrm{S}_{0}$ transition. The band involves a vibrational mode of the ground state, located at 1550 and $1525 \mathrm{~cm}^{-1}$ for $\mathrm{H}_{2} \mathrm{Pc}$ and $\mathrm{ZnPc}$, respectively. Many of the characteristics of amplified emission (AE) are exhibited by this vibronic transition. Excitation scans recorded for the AE band yield greatly enhanced site selectivity compared to what is obtained in normal fluorescence excitation scans.

\section{Introduction}

Although the first absorption spectra of matrix-isolated porphyrins and their synthetic analogues - the phthalocyanines - were reported nearly forty years ago by Bajema et al., ${ }^{1}$ the behaviour of this important class of molecules isolated in the solid rare gases is still not fully characterised. A notable exception is the recent work by Waluk and co-workers on the spectroscopy $^{2}$ and isolation ${ }^{3}$ of porphyrins and related molecules in rare gas matrices. In addition, several groups ${ }^{4}$ have analysed the vibronic structure on the $\mathrm{S}_{1}$ state of several porphyrins and phthalocyanines in Spol'skii matrices.

Laser-induced fluorescence is very well suited for studies of these molecules due to the close match between their strong absorptions in the visible spectral region and the output of tuneable dye lasers. While undertaking such a spectroscopic study of matrix-isolated free-base phthalocyanine $\left(\mathrm{H}_{2} \mathrm{Pc}\right)$ and zinc phthalocyanine $(\mathrm{ZnPc})$, by means of emission-excitation spectra using pulsed dye lasers for excitation, an unusually intense vibronic band was observed with slightly increased laser power. This novel solid state effect is the subject of the present article as it is noteworthy that two previous matrix luminescence studies on the phthalocyanines, one by Bondybey and English ${ }^{5}$ on $\mathrm{H}_{2} \mathrm{Pc}$ and another by Williamson and coworkers ${ }^{6}$ on $\mathrm{ZnPc}$ in $\mathrm{Ar}$ matrices, did not report such an effect. In contrast, stimulated emission has been reported by Sorokin and co-workers ${ }^{7}$ for the closely related molecule chloroaluminium phthalocyanine in solution. Significantly, this was achieved with pulsed ruby laser excitation and within a resonator cavity.

In this paper, the unusual emission recorded primarily for matrix-isolated $\mathrm{H}_{2} \mathrm{Pc}$ and more specifically for $\mathrm{H}_{2} \mathrm{Pc}$ in $\mathrm{N}_{2}$ will be presented. A detailed account of the spectroscopy of $\mathrm{H}_{2} \mathrm{Pc}$ and $\mathrm{ZnPc}$ isolated in a wider range of low temperature solids

\footnotetext{
${ }^{a}$ Department of Chemistry, National University of

Ireland-Maynooth, Co. Kildare, Ireland

${ }^{b}$ Laboratoire de PhotoPhysique Moléculaire du CNRS-Université Paris-Sud, Orsay, France.

E-mail: john.mccaffrey@nuim.ie, claudine.crepin-gilbert@u-psud.fr
}

will be presented elsewhere. ${ }^{8}$ The possible reasons for the different results of the previous laser matrix studies and the present observations will also be discussed.

\section{Experimental}

The spectroscopy of phthalocyanine (Pc) molecules isolated in low temperature solids was undertaken at both the Laboratoire de PhotoPhysique Moléculaire (LPPM) Orsay and the Department of Chemistry, NUI-Maynooth. The oven design used in both experiments was the same, consisting of a solid, stainless steel cylinder into which a hollow screw, containing either free-base $\left(\mathrm{H}_{2} \mathrm{Pc}\right)$ or zinc $(\mathrm{ZnPc})$ phthalocyanines, was fitted. The top of this screw was positioned to emerge at right angles to a $2 \mathrm{~mm}$ opening passing through the length of the cylinder. This opening was connected to the gas inlet line. Resistive heating of the cylinder allowed temperatures of $300{ }^{\circ} \mathrm{C}$ to be reached whereby the Pc vapour was entrained in the flow of the host gas and the mixture deposited on $\mathrm{a} \mathrm{CaF}_{2}$ window at cryogenic temperatures. Gas flows in excess of $10 \mathrm{mmol} / \mathrm{h}$ were required to achieve isolation of monomer Pc. The cryogenic setups have been described previously - the Orsay ${ }^{9}$ system is capable of reaching $6 \mathrm{~K}$ while that at Maynooth ${ }^{10}$ has a base temperature of $12 \mathrm{~K}$. The sample thickness has been measured by an interference method to be in the $250 \mu \mathrm{m}$ region.

The isolation condition of $\mathrm{Pc}$ in the matrix samples formed was monitored with absorption spectroscopy in the red region of the visible spectrum. For this a tungsten lamp source was used. Luminescence (emission-excitation) spectra were recorded using tuneable dye lasers - either a home-built system at Orsay or the Quantel TDL90 system at Maynooth. Both systems are pumped by $10 \mathrm{~Hz}$ nanosecond Q-switched Nd:YAG lasers that have been described previously. ${ }^{9,10}$ Emission was recorded using $i \mathrm{CCD}$ detection with the Andor DH720 system. The absorption and emission spectra were recorded on a $0.6 \mathrm{~m}$ Jobin-Yvon monochromator at Orsay with a resolving power of few $\mathrm{cm}^{-1}$ in the red spectral region, while a pair of lower resolution $(0.3 \mathrm{~m})$ Acton Research 
monochromators was used at Maynooth. No correction for wavelength variations in the sensitivity of the detection system has been made on data presented. Two dyes, DCM and LDS698 were used for laser excitation scans in the wavelength ranges $625-660$ and $665-685 \mathrm{~nm}$, respectively.

\section{Results}

\subsection{Absorption}

The absorption spectra recorded for free-base phthalocyanine $\left(\mathrm{H}_{2} \mathrm{Pc}\right)$ isolated in solid $\mathrm{Ne}, \mathrm{N}_{2}$, and $\mathrm{Ar}$ are shown together on the left hand-side in Fig. 1. The argon spectrum matches that obtained by Bajema et al. ${ }^{1}$ for $\mathrm{H}_{2} \mathrm{Pc} / \mathrm{Ar}$ while the $\mathrm{Ne}$ and $\mathrm{N}_{2}$ spectra do not appear to have been previously published. The Ar data also compares well with the higher resolution absorption spectra reported more recently by Kador and co-workers $^{11}$ and clearly corresponds to well-isolated guest $\mathrm{H}_{2} \mathrm{Pc}$ molecules. The two principal features in the $\mathrm{H}_{2} \mathrm{Pc}$ spectra recorded in all of the matrices correspond to absorptions of the split $S_{1}$ state, frequently labelled the $Q_{X}$ and $Q_{Y}$ states ${ }^{12}$ in order of increasing energy. The shapes of the bands differ markedly between the solids shown with very structured features present in $\mathrm{N}_{2}$ and a broad unstructured band present in Ne. Ar presents what seems to be the simplest spectrum. However, as revealed in laser excitation spectroscopy, the widths of the bands in all matrices arise from the occupancy of $\mathrm{H}_{2} \mathrm{Pc}$ in multiple sites. It is only in $\mathrm{N}_{2}$ that some of the absorption lines correspond to individual resolved sites. In this matrix, the absorption linewidths $\left(4-5 \mathrm{~cm}^{-1}\right)$ are quite close to the resolving power of the recording monochromator (approx. $2 \mathrm{~cm}^{-1}$ ).

\subsection{Fluorescence}

Representative emission spectra recorded for $\mathrm{H}_{2} \mathrm{Pc}$ in the three aforementioned solids are shown on the right in Fig. 1. The features in Ar closely match those reported by Bondybey and

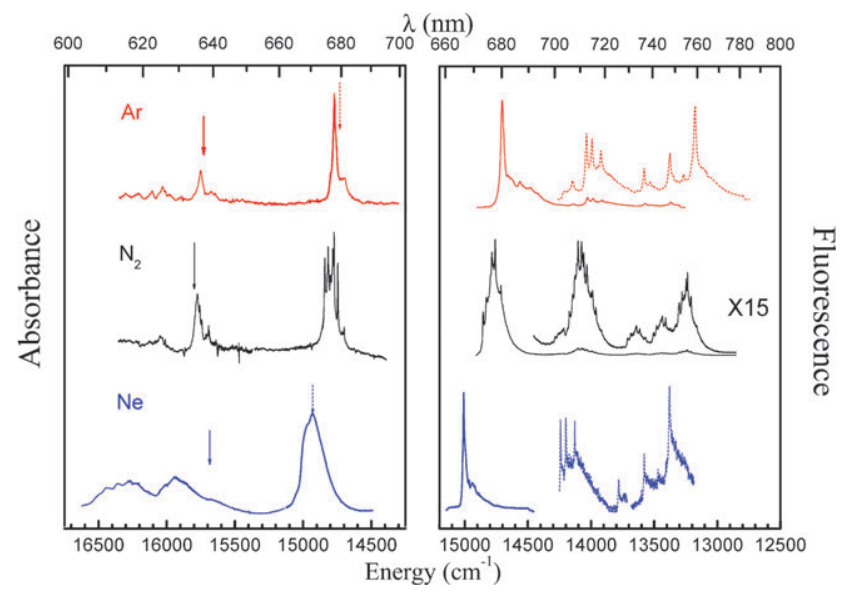

Fig. 1 Absorption spectra recorded for free-base phthalocyanine $\left(\mathrm{H}_{2} \mathrm{Pc}\right)$ isolated in solid $\mathrm{Ne}, \mathrm{N}_{2}$, and $\mathrm{Ar}$ are shown on the left hand side. Emission spectra recorded for $\mathrm{H}_{2} \mathrm{Pc}$ isolated in the three matrices are shown on the right. The arrows shown on the left with the absorption spectra indicated the laser excitation wavelengths used in producing the main emission spectra. $\mathrm{Q}_{\mathrm{X}}$ excitation is represented by broken arrows while the solid arrows depict higher energy excitation.
English. ${ }^{5}$ In the spectrum it is evident that the $0-0$ transition at $677 \mathrm{~nm}$ dominates the emission intensity by more than a factor of 10 . This is consistent with the results of measurements made in low temperature molecular beams ${ }^{13-15}$ in which the $0-0$ transition was found to carry most of the Franck-Condon intensity. The next most intense band in the Ar spectrum is that centered at $756 \mathrm{~nm}$. The emission bands in Fig. 1 are assigned to transitions from $v^{\prime}=0$ in the excited $\mathrm{Q}_{\mathrm{X}}$ state to various vibrational levels $v^{\prime \prime}$ in the ground $\mathrm{S}_{0}$ electronic state. The vibronic progression in emission is quite similar for the three hosts shown in Fig. 1. The frequencies of the main vibronic transitions are reported in Table 1. The emission produced with laser excitation of the $\mathrm{Q}_{\mathrm{Y}}$ state corresponds to the $\mathrm{Q}_{\mathrm{X}} \rightarrow \mathrm{S}_{0}$ transition and exhibits, as shown in Fig. 1, the same vibronic structure. While the $\mathrm{Q}_{\mathrm{X}}$ excitation allows site selective excitation yielding narrow emission bands, $Q_{Y}$ excitation provides much poorer site selection, as evidenced by a broadening of emission bands in Ar or an additional structure involving multiple sites in nitrogen. Moreover, the extensive overlap of sites that occurs in nitrogen also produces greater intensity of the $710 \mathrm{~nm}$ bands evident in Fig. 1. The emission spectra recorded for $\mathrm{ZnPc}$ (not shown) are broadly similar to those presented in Fig. 1 for $\mathrm{H}_{2} \mathrm{Pc}$. Indeed both systems show strong similarities to the emission recorded in Shpol'skii matrices. ${ }^{16,17}$

\subsection{Amplified emission}

While optimising the emission signal with the iCCD camera operating in real time, the intensity of the exciting laser was increased with a quite unexpected result. To illustrate the observed effect, a summary of the changes that were recorded for $\mathrm{H}_{2} \mathrm{Pc}$ in solid $\mathrm{N}_{2}$ is presented in Fig. 2. In this Figure the normal fluorescence, described in the previous section, is shown by the lower, dashed trace. Shown above this, by the solid trace, is the emission recorded by increasing the laser power from tens of $\mu \mathrm{J} /$ pulse to hundreds of $\mu \mathrm{J} /$ pulse. It is very evident that the strongest (other than the $0-0$ band) emission band at $755.5 \mathrm{~nm}$ has gained enormously in intensity while the others have remained unchanged or diminished slightly. Moreover, it is immediately evident that the linewidth of this emission band reduces considerably when its intensity increases as shown on the upper right panel of Fig. 2. Under this condition the width of the band approaches that of the exciting laser, decreasing from approximately $8 \mathrm{~cm}^{-1}$ to $2 \mathrm{~cm}^{-1}$ and thereby reaching the resolving limit of the emission monochromator.

Shown in the inset on the left of Fig. 2 are the emission decay curves recorded for the $755.5 \mathrm{~nm}$ emission band using selected laser excitation intensities. With low laser power, the long-lived decay (stars) is found to have a lifetime of around $12 \mathrm{~ns}$. With higher laser power the intense emission (squares) clearly follows the temporal profile of the laser pulse (triangles). From the spectral and temporal behaviour observed on this band, we conclude that the $755.5 \mathrm{~nm}$ emission is being amplified when the excitation laser intensity exceeds a certain value. It corresponds to reaching a threshold value in the population inversion between $v^{\prime}=0$ of the excited $\left(\mathrm{Q}_{\mathrm{X}}\right)$ electronic state and $v^{\prime \prime}=1$ of a specific vibrational mode in 
Table 1 Energies $\left(\mathrm{cm}^{-1}\right)$ of the main $\mathrm{S}_{1}\left(\mathrm{Q}_{\mathrm{X}}\right) \leftrightarrow \mathrm{S}_{0}$ vibronic transitions of $\mathrm{H}_{2} \mathrm{Pc}$ isolated in $\mathrm{Ne}$, Ar and $\mathrm{N}_{2}$ matrices. The $0-0$ values were determined from absorption spectra. The emission values quoted correspond to the main bands observed as the triplet set around $710 \mathrm{~nm}$ and the most intense band around $755 \mathrm{~nm}$ that exhibits AE. The emission values in parenthesis are frequency shifts from the $0-0$ transition listed in column 2

\begin{tabular}{|c|c|c|c|c|c|}
\hline $\mathrm{H}_{2} \mathrm{Pc}$ & $0-0$ & & & & $\mathrm{AE}$ \\
\hline \multirow{5}{*}{$\mathrm{N}_{2}$ (7 main sites) } & 14856 & 14169 (687) & $14126(730)$ & 14057 (799) & $13302(1554)$ \\
\hline & 14817 & $14130(687)$ & $14086(731)$ & $14016(801)$ & 13264 (1553) \\
\hline & 14799 & $14111(688)$ & $14068(731)$ & 13997 (802) & $13249(1550)$ \\
\hline & 14787 & $14102(687)$ & $14058(731)$ & 13987 (802) & $13236(1551)$ \\
\hline & $\begin{array}{l}14762 \\
14715^{a}\end{array}$ & $14075(687)$ & $14032(730)$ & $13960(802)$ & $13210(1552)$ \\
\hline
\end{tabular}

Ne (continuum of sites) 14 954-to-14 90514 266-to-(688) $14217 \quad 14$ 223-to-(731) 1417414 153-to-(801) 1410413402 (1552)-to-13 352 (1553)

${ }^{a}$ Weak emission, no AE observed.

the ground electronic state. For the mode involved, spontaneous emission is amplified by stimulated emission once the threshold value is exceeded. We will refer to this process in a general way as amplified emission (AE).

Although not presented in detail in this article, amplified emission has also been observed for $\mathrm{H}_{2} \mathrm{Pc}$ isolated in $\mathrm{Ne}, \mathrm{Ar}$, $\mathrm{Kr}$ but not in Xe hosts. When observed, AE always appears on the same vibronic transition involving a vibrational mode around $1550 \mathrm{~cm}^{-1}$ (Table 1). In xenon it has been determined that the normal fluorescence decay time is considerably shorter than in the other hosts, indicating that an efficient nonradiative decay mechanism is present in this solid. The likely origin of this effect lies in the external heavy atom effect which increases the probability of intersystem crossing from the excited singlet $S_{1}$ state to the lower lying spin triplet state, $\mathrm{T}_{1}$ state. Thus another relaxation mechanism is competing for the fluorescence and thereby reduces the chance of the excited singlet state population reaching the threshold condition. In the case of $\mathrm{H}_{2} \mathrm{Pc} / \mathrm{Ne}$, a very efficient hole-burning phenomenon is observed in the $0-0 \mathrm{Q}_{\mathrm{X}} \leftarrow \mathrm{S}_{0}$ absorption band with even weak dye laser excitation [data not shown]. As the excited family of sites disappears during excitation, AE is not sustainable for long periods in this solid. The results obtained in the zinc phthalocyanine $(\mathrm{ZnPc})$ system closely mirror those presented herein for the free-base system. Thus, a single mode is amplified in $\mathrm{ZnPc}$ at $1525 \mathrm{~cm}^{-1}$ equivalent to the $1550 \mathrm{~cm}^{-1}$

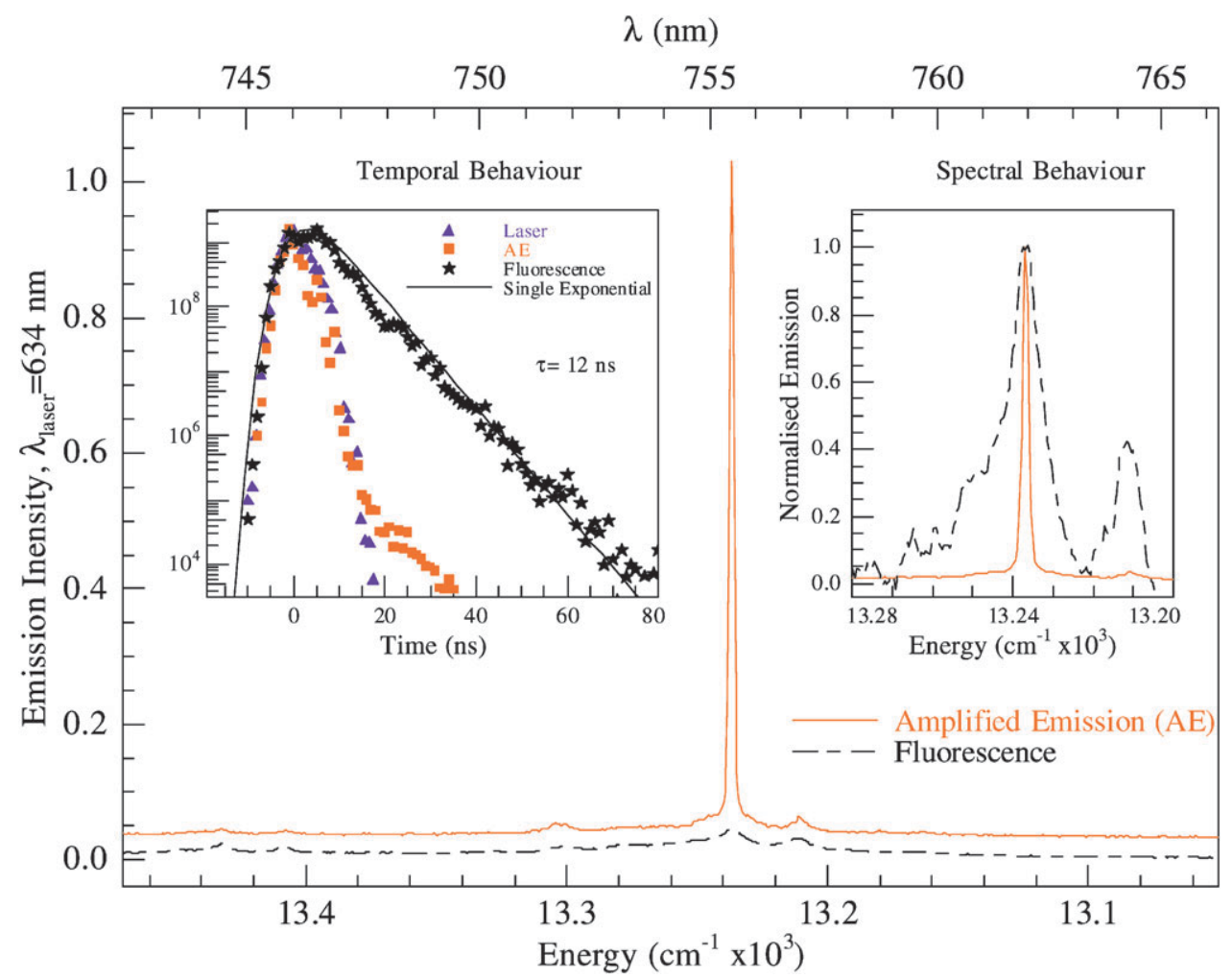

Fig. 2 Variations in the characteristics of the $755.5 \mathrm{~nm}$ emission of $\mathrm{H}_{2} \mathrm{Pc} / \mathrm{N}_{2}$ system with laser intensity. The lower trace presents the normal emission (fluorescence) produced with lower laser power $(10 \mu \mathrm{J} /$ pulse). The upper trace shows the dramatic increase in the intensity of the $755.5 \mathrm{~nm}$ emission with the use of approximately $100 \mu \mathrm{J} /$ pulse. The inset on the right shows details of the lineshape changes on the $755.5 \mathrm{~nm}$ band under both low and high pulse energies. Temporal profiles of the $755.5 \mathrm{~nm}$ emission decay curves are shown in the inset on the left hand side. 
mode of $\mathrm{H}_{2} \mathrm{Pc}$. As found for $\mathrm{H}_{2} \mathrm{Pc}, \mathrm{AE}$ is observed for $\mathrm{ZnPc}$ in all the solids studied except xenon.

\subsection{Threshold for amplified emission}

A threshold in the dependence of the intensity of the amplified emission on the excitation laser intensity is clearly observed under both $\mathrm{Q}_{\mathrm{X}}$ and $\mathrm{Q}_{\mathrm{Y}}$ excitation. Threshold data is shown in Fig. 3 for the dominant site emission in $\mathrm{H}_{2} \mathrm{Pc} / \mathrm{N}_{2}(755.5 \mathrm{~nm})$ produced with excitation at $634 \mathrm{~nm}\left(\mathrm{Q}_{\mathrm{Y}}\right.$ excitation). The band exhibiting amplification at $755.5 \mathrm{~nm}$ is represented in Fig. 3 by the filled circles. For comparison, normal fluorescence from the same site is shown in Fig. 3 by the emission bands at 744.4 and $676.3 \mathrm{~nm}$ (the $0-0$ band) which were not observed to be amplified. As shown in the lower portion of the plot, the growth in the intensities of these two emission bands track the $755.5 \mathrm{~nm}$ band up to a specific value of the exciting laser intensity. Beyond this value, the intensity of $755.5 \mathrm{~nm}$ band rapidly increases while the fluorescence intensity remains unchanged on the scale shown. To identify the point beyond

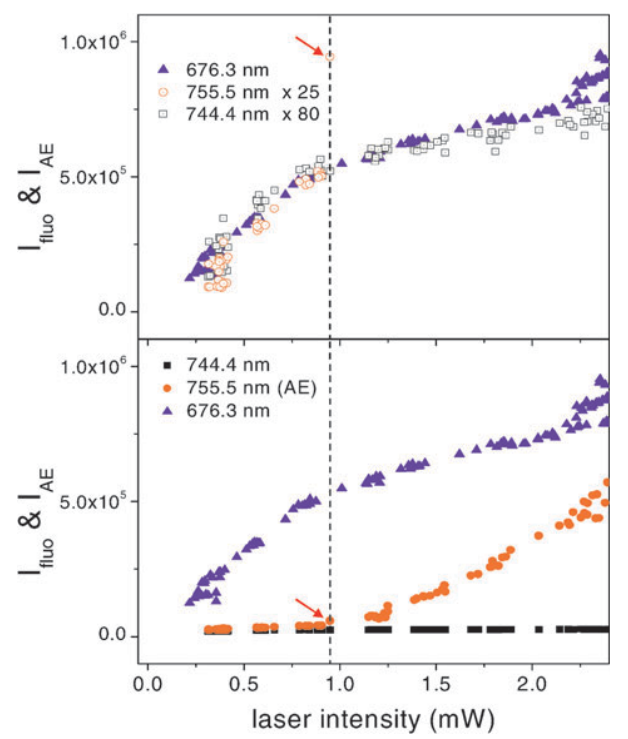

Fig. 3 Threshold curves measured for the dominant site emission of free-base phthalocyanine $\left(\mathrm{H}_{2} \mathrm{Pc}\right)$ isolated in solid $\mathrm{N}_{2}$. The data were recorded by monitoring the emission intensities at the indicated wavelengths while varying the laser excitation intensity at $634 \mathrm{~nm}$. In the lower panel the wavelengths selected $676.3,744.4$ and $755.5 \mathrm{~nm}$ correspond to $0-0$ emission of the $\mathrm{Q}_{\mathrm{X}} \rightarrow \mathrm{S}_{0}$ transition at $14787 \mathrm{~cm}^{-1}$, fluorescence of a vibronic band at $13433 \mathrm{~cm}^{-1}$ and the amplified emission band at $13236 \mathrm{~cm}^{-1}$, respectively. In the upper panel the same three bands are shown (open symbols) but the intensities of the bands at 755.5 and $744.4 \mathrm{~nm}$ have been multiplied by factors of 25 and 80 , respectively, to match the intensity of the $0-0$ band at $676.3 \mathrm{~nm}$. It is evident at low pulse power that the intensities of the three emission bands can be overlaid exactly, demonstrating that below threshold all three emission bands follow the same dependence on excitation intensity. However, the sudden change in slope of the $755 \mathrm{~nm}$ band, depicted by the single open circle at the top of the figure (shown by the arrows in the two panels) and indicated by dashed vertical line, indicates the onset of amplified emission (AE) from normal fluorescence. This point, identified to be $0.95 \mathrm{~mW}$, is the threshold for the generation of $\mathrm{AE}$ on the $13236 \mathrm{~cm}^{-1}$ band which is $1551 \mathrm{~cm}^{-1}$ from the band origin. which amplified emission takes over from fluorescence, the three sets of data are shown on a larger scale in the upper portion of the plot. The threshold value is indicated in Fig. 3 by the vertical dashed line at approximately $0.95 \mathrm{~mW}(95 \mu \mathrm{J} /$ pulse). The intensity of non-amplified emission follows the same behaviour as the amplified band before the threshold, but the slope slightly decreases beyond this point. When the amplification of one vibronic emission occurs, the corresponding specific transition becomes a preferential relaxation path (open circle).

The threshold condition for amplified emission is given ${ }^{18}$ by the expression,

$$
\frac{N_{\mathrm{th}}}{V}=\frac{8 \pi \tau \Delta \nu}{\lambda^{2} l \phi} n^{2}
$$

in terms of the number density of the excited state molecules per cubic centimetre. In this equation $\tau$ is the radiative lifetime of the excited $S_{1}\left(Q_{X}\right)$ state (assumed to be equal to the measured lifetime, $12 \mathrm{~ns}), \Delta \nu$ is the emission linewidth (8 $\left.\mathrm{cm}^{-1}, 240 \mathrm{GHz}\right), \lambda$ is the wavelength $(755.5 \mathrm{~nm})$ and $\phi$ is the emission quantum yield of this vibronic transition $(\phi=0.05)$. $l$ is the sample pathlength $(0.025 \mathrm{~cm})$ and $n$ is the index of refraction (1.35) of the medium. Making substitutions for the photophysical parameters measured for the $\mathrm{H}_{2} \mathrm{Pc} / \mathrm{N}_{2}$ transition, given in parenthesis, we obtain a threshold value of $2 \times$ $10^{16} \mathrm{~cm}^{-3}$. While this is a very large value for gas phase conditions, it is at least 2 orders of magnitude less than the concentration of the ground state molecules isolated in the solid. Accordingly, it has been observed that AE does not occur in very dilute samples in which this threshold cannot be reached with similar excitation powers. In contrast, AE is easily observed for slightly higher concentration, as shown in the example of Fig. 4 where AE has been recorded on most of the families of sites in $\mathrm{N}_{2}$.

\subsection{Excitation spectra and improved site resolution with amplified emission}

Fig. 4 shows a two-dimensional (2-D) excitation/emission plot of the AE observed for free-base phthalocyanine $\left(\mathrm{H}_{2} \mathrm{Pc}\right)$ isolated in solid $\mathrm{N}_{2}$. The plot depicts the variety of sites detected with excitation in the $0-0$ region of the $\mathrm{Q}_{\mathrm{X}} \leftarrow \mathrm{S}_{0}$ transition while monitoring the $\mathrm{AE}$ band centered around $755 \mathrm{~nm}$. Shown on the right hand side and on the upper part of the figure are characteristic excitation and emission slices recorded for normal fluorescence (dotted lines) and under AE conditions. The increased resolution on an individual site is evident in both emission and excitation spectra. However, it is especially evident in the excitation scans shown on the right hand side where the lineshapes change from a featureless band (dotted line) to a highly structured band (solid trace) exhibiting what appears to be a sharp zero-phonon line and a structured side-band. Other examples of excitation spectra recorded under AE conditions are depicted by traces $\mathrm{b}-\mathrm{e}$ in Fig. 5 and compared with normal fluorescence excitation scans recorded with the pump laser intensity below threshold (a). Clearly excitation spectra recorded for the AE signal provide greatly enhanced spectroscopic information over what can be 


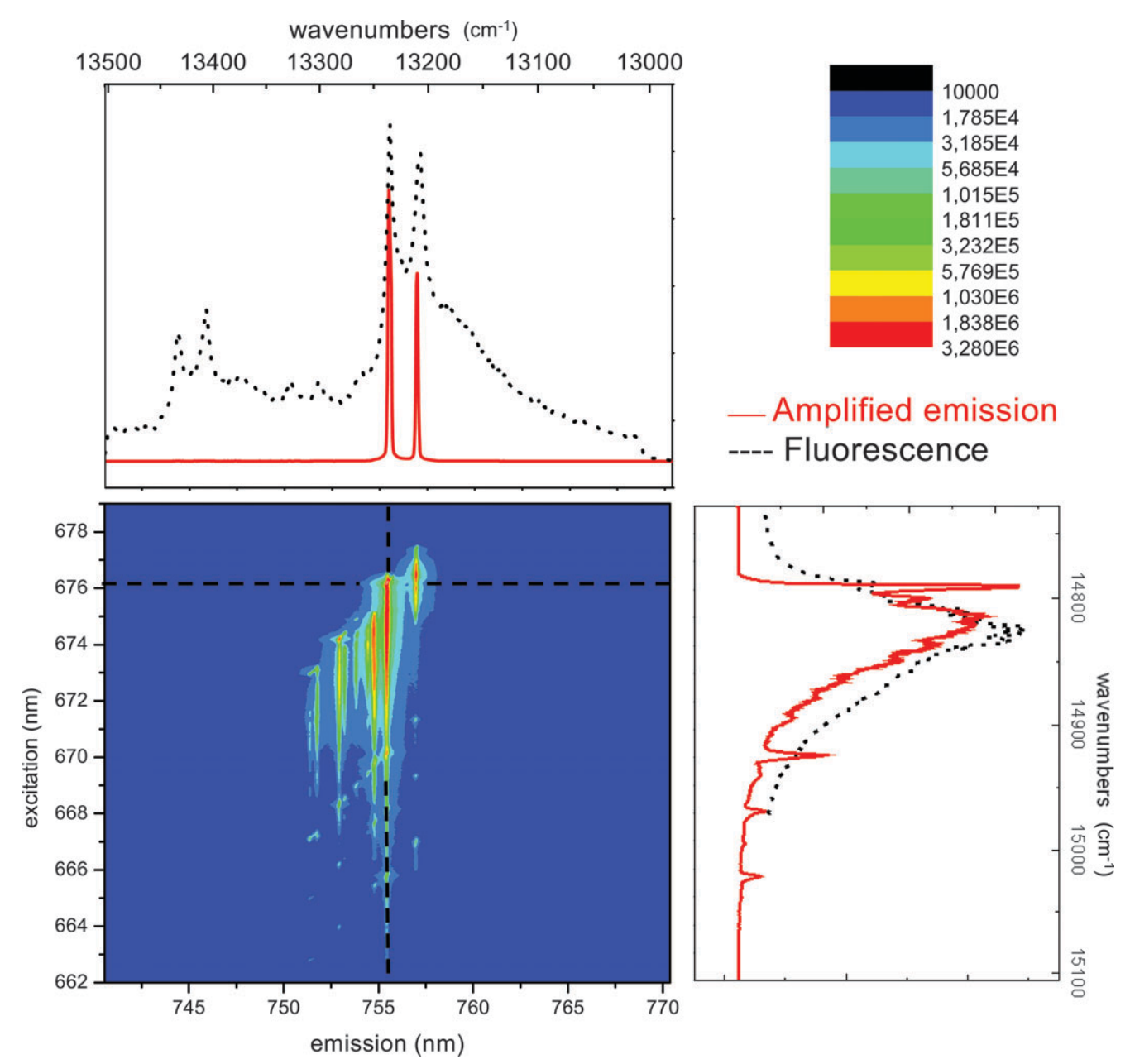

Fig. 4 2-D excitation-emission plots of the AE observed for free-base phthalocyanine $\left(\mathrm{H}_{2} \mathrm{Pc}\right)$ isolated in solid $\mathrm{N}_{2}$. The data were recorded by monitoring emission with iCCD detection while scanning the tuneable dye laser used for excitation at high intensity. The variety of sites is indicated in the central portion of the plot. Characteristic excitation and emission slices are shown on the right hand side and on the upper parts of the Figure, respectively, corresponding to the positions in emission and excitation given by the black dots on the 2D plot. Particularly noteworthy is the improved resolution of the AE excitation/emission scans compared with that recorded from the conventional fluorescence signal shown by the dotted lines at the same emission-excitation wavelengths.

extracted from either conventional fluorescence excitation or absorption spectroscopy.

The sharp resolved lines present in the AE excitation scan in $\mathrm{N}_{2}$ are shifted by 130,178 and $228 \mathrm{~cm}^{-1}$, as shown in Fig. 5, from the $0-0$ transition. They correspond to a vibronic progression on the $\mathrm{Q}_{\mathrm{X}} \leftarrow \mathrm{S}_{0}$ transition that has been identified in the excitation scans of the molecule in low temperature matrices ${ }^{5,17}$ and molecular beams. ${ }^{15}$ The AE excitation spectra (b-e) shown in Fig. 5 depict the range of behaviour exhibited by the different sites for the $\mathrm{QX}_{\mathrm{X}}$ state of $\mathrm{H}_{2} \mathrm{Pc}$ in $\mathrm{N}_{2}$. Most representative is trace (b) which exhibits a clearly identifiable zero-phonon line (ZPL) for the $0-0$ transition with a more intense phonon sideband (PSB). Due to the strength and the width of the latter bands, excitation of the ZPL of a blue site simultaneously excites the PSBs of the red sites. As a result, $\mathrm{AE}$ is then observed in all the sites as shown in Fig. 4. Strong phonon sidebands are also observed in the excitation spectra recorded in $\mathrm{Ar}$ and $\mathrm{Kr}$ matrices. Indeed for some sites the ZPL can even be absent. In solid Ar, AE appears mainly at only one frequency assigned to a vibronic transition of the molecule occupying the main site: the threshold of $\mathrm{AE}$ is much easier to reach for this specific site which can be efficiently populated in the excited state through the phonon band. In contrast, excitation spectra of $\mathrm{AE}$ in solid $\mathrm{Ne}$ are dominated by the $0-0$ transition line. There is a continuum of equally occupied sites (see absorption spectrum in Fig. 1) and the AE frequency follows the excitation frequency all along the excitation in the broad absorption band. It seems that in this "soft" solid there is no preferential site geometry-a possible reason for the unusual behaviour sometimes observed ${ }^{19}$ with the use of neon as a host material. Thus, the observation of AE should be useful to determine the number of main families of sites in a given host and to underline the differences between the sites occupied in these hosts.

\section{Discussion}

The essentials of the AE observed in this work can be described with the energy level scheme shown in Fig. 6. It comprises of the ground state [Level $1, \mathrm{~S}_{0}\left(v^{\prime \prime}=0\right)$ ], which is 


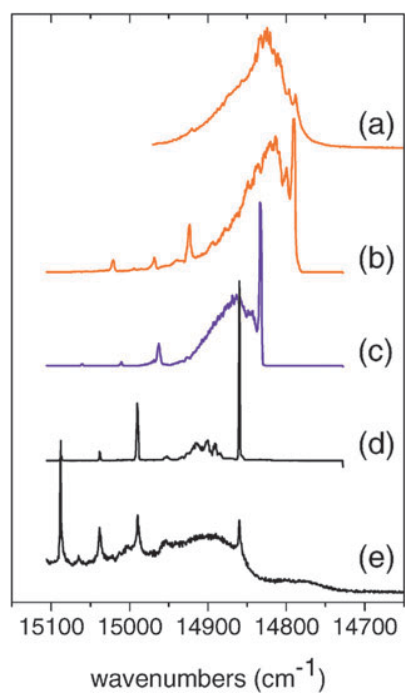

Fig. 5 A selection of laser excitation scans recorded with AE, involving the $1550 \mathrm{~cm}^{-1}(\mathrm{~b}-\mathrm{d})$ and the $730 \mathrm{~cm}^{-1}$ (e) modes, for a variety of sites occupied by free-base phthalocyanine $\left(\mathrm{H}_{2} \mathrm{Pc}\right)$ isolated in solid $\mathrm{N}_{2}$. The emission bands monitored are at $13236 \mathrm{~cm}^{-1}(\mathrm{a}-\mathrm{b})$, $13279 \mathrm{~cm}^{-1}$ (c), $13302 \mathrm{~cm}^{-1}$ (d) and $14126 \mathrm{~cm}^{-1}$ (e). For the purpose of comparison a conventional fluorescence excitation scan is shown in (a). The spectra recorded range from a site with moderate electron-phonon coupling (b) to one with vanishing small coupling (d). The sharp features evident in the four AE scans (b)-(e), correspond to the low frequency vibrational modes in the $\mathrm{Q}_{\mathrm{X}}$ excited state.

the only one populated at $10 \mathrm{~K}$ before laser excitation, the pumped state, the upper level of the AE [Level 2, $\mathrm{Q}_{\mathrm{X}}\left(v^{\prime}=0\right)$ or in general Q] and the lower level of AE [Level 3, $\mathrm{S}_{0}\left(v^{\prime \prime}=1\right.$ of a specific mode)] i.e., at $1550 \mathrm{~cm}^{-1}$ above the vibrationless ground state. In general we have a 4-level scheme because a Level $2^{\prime}$ is usually involved. As indicated in Fig. 6, this is either a vibrationally excited level of $\mathrm{Q}_{\mathrm{X}}$ or the $\mathrm{Q}_{\mathrm{Y}}$ electronic state. For AE to occur, an efficient route for populating Level 2 is required. This is provided by very efficient pumping with pulsed laser excitation of an electronic transition having a huge extinction coefficient $-\varepsilon=162000 \mathrm{~cm}^{-1} / \mathrm{M}$ at $698.5 \mathrm{~nm}$ for $\mathrm{H}_{2} \mathrm{Pc}$ in chloronaphthalene. ${ }^{20}$ For indirect excitation via the $\mathrm{Q}_{\mathrm{Y}}$ state (Level $2^{\prime}$ ) an efficient relaxation to Level 2 is required. This is indeed the case, because the only emission that has been observed with $\mathrm{Q}_{\mathrm{Y}}$ is relaxed, originating from $v^{\prime}=0$ in the $\mathrm{Q}_{\mathrm{x}}$ state. For the main sites in $\mathrm{N}_{2}$, we have not observed any Stokes shift on the $0-0 \mathrm{QX}_{\mathrm{x}} \leftrightarrow \mathrm{S}_{0}$ transition so with direct pumping of $v^{\prime}=0$ in the $\mathrm{Q}_{\mathrm{X}}$ state, there is, in this matrix, only a single Level 2. Consistent with this, AE seems easier to reach by pumping $\mathrm{Q}_{\mathrm{X}}$ than $\mathrm{Q}_{\mathrm{Y}}$. Finally, Level 3 corresponds to a non-thermally populated level at $1550 \mathrm{~cm}^{-1}$ so that the inversion population on the (2) $\rightarrow$ (3) transition is easily obtained.

In order to maintain the population inversion during the course of the 10 ns laser pump pulse, the vibrational Level 3 must rapidly relax to vibrational levels of lower energy. Level 2 of $\mathrm{H}_{2} \mathrm{Pc}$ has an observed lifetime of $12 \mathrm{~ns}$ in $\mathrm{N}_{2}$ solid, indicating that Level 3 must decay more quickly than this. From DFT calculations ${ }^{21}$ on $\mathrm{ZnPc}$, the $1525 \mathrm{~cm}^{-1}$ vibrational mode (equivalent to $1550 \mathrm{~cm}^{-1}$ mode in $\mathrm{H}_{2} \mathrm{Pc}$ ) involves out-ofphase stretching of the $\mathrm{C}-\mathrm{N}-\mathrm{C}$ bridges on the inner ring of $\mathrm{Pc}$.

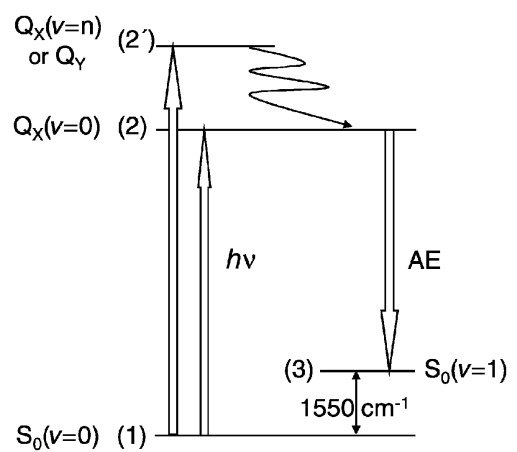

Fig. 6 A schematic energy level diagram of the states involved in the amplified emission of the free base phthalocyanine. The effect of AE can be produced with $0-0$ excitation (2) of the $\mathrm{Q}_{\mathrm{X}}$ state or with higher energy excitation $\left(2^{\prime}\right)$ into either vibrationally excited levels of this state or into the $\mathrm{Q}_{\mathrm{Y}}$ state.

Thus it is expected that this mode can relax very quickly towards low frequency modes. Indeed the measured fluorescence bandwidth of few wavenumbers is consistent with typical vibrational lifetimes in the picosecond range.

As already mentioned, large population can be very efficiently pumped into the available levels 2 with pulsed laser excitation of phthalocyanines. These dye materials were examined by Sorokin ${ }^{7}$ as good candidates to generate stimulated emission around $750 \mathrm{~nm}$. With this characteristic, $\mathrm{H}_{2} \mathrm{Pc}$ and $\mathrm{ZnPc}$ seem ideal molecules to observe the AE process in low temperature matrices. From eqn (1) it is evident that threshold will be reached most easily for strongly allowed transitions (short $\tau$ ) with narrow linewidths (small $\Delta \nu$ ) and for high fluorescence quantum yields. All of the parameters in the threshold equation, except the pathlength, are favoured in the case of $\mathrm{H}_{2} \mathrm{Pc}$ emission in low temperature matrices. Especially favourable is the narrow emission linewidth $\left(\sim 8 \mathrm{~cm}^{-1}\right)$ on a fully allowed electronic transition Arising from these observations, we predict that $\mathrm{AE}$ should also be possible for these dye molecules isolated in Shpol'skii matrices when excited with short pulse lasers.

Another key factor in generating amplified emission is the nature of the laser excitation. Thus to achieve the threshold population in the excited state, it is essential that a temporally compact excitation is used such as that provided by the nanosecond pulse of a Q-switched Nd:YAG laser. In this regard, when we used a low intensity laser pulse, only normal fluorescence was observed. Similarly, if a cw laser is used, the threshold condition will not be attained. We surmise that it was for these reasons that $\mathrm{AE}$ emission was not observed in any of the previous laser studies of the matrix-isolated phthalocyanines. Thus, Bondybey and English ${ }^{5}$ used a low power pulsed nitrogen laser, while in the more recent study of Williamson and co-workers, ${ }^{6}$ a dye laser pumped by a cw $\mathrm{Ar}^{+}$laser was employed. The detailed spectroscopic work that have been done on free-base and metallo-phthalocyanines, by Huang et al. ${ }^{17}$ also utilised a dye laser pumped by a cw $\mathrm{Ar}^{+}$ laser. With cw excitation the threshold population will not be reached and only normal fluorescence will be observed. An additional parameter that renders this effect difficult to observe in low temperature matrices is the short path length 
$(l=250 \mu \mathrm{m})$ of these thin film samples. In comparison, production of amplified emission in the gas phase ${ }^{22}$ requires the use of cells whose length are typically tens of centimetres.

The next question that must be addressed in the observations presented in this article is why does only a single mode exhibit amplification? The answer to this question is also to be found in eqn (1). Thus for molecular emission involving several vibrational transitions, the threshold will be reached most easily for the mode with the largest Franck-Condon factor in order to optimise $\phi$. In other words, it is to be expected that the most intense emission band will reach threshold first. It will be remembered as shown in Fig. 1, the $0-0$ band (around $676 \mathrm{~nm}$ ) is much stronger than the vibronic band around $755 \mathrm{~nm}$ which becomes amplified. However, there is extensive spectral overlap of absorption and emission bands for the $0-0$ transition. Hence, this transition will suffer from competitive absorption and accordingly will not reach threshold. Moreover, the threshold in this specific case is much higher than that given by eqn (1) because of the large population in the lower level, i.e., the vibrationless ground state.

In the previous studies of phthalocyanine in solution, ${ }^{7}$ isolated in organic solids ${ }^{17,23}$ or matrices ${ }^{5,6}$ the transition involving the vibrational mode around $1550 \mathrm{~cm}^{-1}$ is always either one of the more intense bands or the most intense band in emission spectra. The stimulated emission observed by Sorokin et al. ${ }^{7}$ for AlPc in ethanol was also on this vibronic transition. Other vibrational modes appear in the emission spectrum with intensities similar to the $1550 \mathrm{~cm}^{-1}$ mode (Fig. 1). In fact, some experiments done on quite concentrated $\mathrm{H}_{2} \mathrm{Pc}$ samples with high laser intensities have shown that $\mathrm{AE}$ is also possible on other vibronic transitions. Thus AE has been observed in $\mathrm{N}_{2}$ solid, when exciting specific sites involving modes at 687 and $730 \mathrm{~cm}^{-1}$ (a related excitation spectrum is shown in Fig. 5e). Further experiments are currently underway and complete details of the spectroscopy of $\mathrm{ZnPc}$ and $\mathrm{H}_{2} \mathrm{Pc}$ isolated in a more extensive range of low temperature solids will be presented elsewhere. ${ }^{8}$

\section{Conclusions}

The luminescence spectroscopy of phthalocyanines embedded in low temperature solids has revealed the phenomenon of amplified emission under modest conditions of pulsed laser excitation. The possibility an iCCD camera provides of very efficiently collecting high resolution 2-D excitation/emission scans has been central to the discovery of this unexpected phenomenon. In addition to the huge increase in intensity, the lineshape of the AE band narrows and its decay time shortens so that it matches the Q-switch laser used for excitation. The photophysical characteristics conducive to this effect are analysed and it is concluded the key conditions are (1) the large absorption strengths of these dye materials, (2) the spectrally narrow emission lines that these molecules exhibit and (3) the use of a Q-switched excitation laser. Accordingly, we predict that AE should also be observable for these molecules isolated in other solid materials that produce narrow linewidths such as Shpol'skii matrices.

Excitation scans recorded for the AE mode yield greatly enhanced site selectivity compared to what is obtained in normal fluorescence excitation scans. This behaviour stems from the increased resolution of individual sites whose lineshape change from featureless fluorescence excitation bands to a highly structured AE excitation band. The very well-resolved spectra available in AE excitation scans allows detailed exploration of site occupancies in a given solid and from one solid to another. Differences in matrix sites have been observed and analysed by Waluk and co-workers ${ }^{3}$ in the case of porphyrins and porphycenes which are very similar molecules. The observations of AE, made in the present study with only moderate laser excitation intensities, should allow new insights into site effects and site selectivity in future studies of solid state spectroscopy.

\section{Acknowledgements}

The assistance of Dr Michel Broquier and Dr Pierre Çarçabal with the design of the oven used for vaporisation of the Pcs is gratefully acknowledged as are fruitful discussions with $\mathrm{Dr}$ Jean Pierre Galaup, Dr Serguei Arabei and Dr Vadim Alekseev. This work was supported by the Ulysses France-Ireland research exchange grant (2006) and the Science Foundation Ireland (SFI), Research Frontiers Programme (06/RFP/ CHP012) Grant.

\section{References}

1 L. Bajema, M. Gouterman and B. Meyer, J. Mol. Spectrosc., 1968, 27, 225.

2 A. Starukhin, A. Shulga and J. Waluk, Chem. Phys. Lett., 1997, 272, 405.

3 A. Kyrychenko and J. Waluk, J. Chem. Phys., 2005, 123, 064706 and references cited therein.

4 S. M. Arabei, J. P. Galaup, K. N. Solovyov and V. F. Donyagina, Chem. Phys., 2005, 311, 307; S. M. Arabei, K. N. Solov'ev and A. E. Turkova, Optics and Spectroscopy, 1996, 80, 379.

5 V. E. Bondybey and J. H. English, J. Am. Chem. Soc., 1979, 101, 3446.

6 B. J. Prince, B. E. Williamson and R. J. Reeves, J. Lumin., 2001, 93, 293.

7 P. P. Sorokin and J. R. Lankard, IBM Journal, 1966, 10, 162; also P. P. Sorokin, J. R. Lankard, E. C. Hammond and V. L. Moruzzi, IBM J. Res. Dev., 1967, 11, 130.

8 N. Dozova, C. Murray, J. G. McCaffrey, N. Shafizadeh, and C. Crépin, manuscript in preparation.

9 C. Gée, A. Cuisset, L. Divay and C. Crépin, J. Chem. Phys., 2002, 116, 4993

10 M. A. Collier and J. G. McCaffrey, J. Chem. Phys., 2005, 122, 184507.

11 P. Geissinger, L. Kador and D. Haarer, Phys. Rev. B, 1996, 53, 4356.

12 In the literature of the porphyrins and phthalocyanines the electronic states $\mathrm{S}_{0}, \mathrm{~S}_{1}, \mathrm{~S}_{2}, \mathrm{~S}_{3}$ and $\mathrm{S}_{4}$ of the $D_{2 \mathrm{~h}}$ symmetry free-base molecules are frequently designated $\mathrm{G}_{2} \mathrm{Q}_{\mathrm{X}}, \mathrm{Q}_{\mathrm{Y}}, \mathrm{B}_{\mathrm{X}}$ and $\mathrm{B}_{\mathrm{Y}}$, respectively. In addition, the symbols $\mathrm{S}_{1}{ }^{\mathrm{X}}$ and $\mathrm{S}_{1}{ }^{\mathrm{Y}}$ have also used to indicate $\mathrm{Q}_{\mathrm{X}}$ and $\mathrm{Q}_{\mathrm{Y}}$ levels, respectively in the split the $\mathrm{S}_{1}$ state of the free-base phthalocyanine $\left(\mathrm{H}_{2} \mathrm{Pc}\right)$.

13 P. S. H. Fitch, C. A. Haynam and D. H. Levy, J. Chem. Phys., 1980, 73, 1064; also P. S. H. Fitch, C. A. Haynam and D. H. Levy, J. Chem. Phys., 1981, 74, 6612.

14 J. A. Menapace and E. R. Bernstein, J. Chem. Phys., 1987, 87, 6877.

15 F. L. Plows and A. C. Jones, J. Mol. Spectrosc., 1999, 194, 163.

16 R. I. Personov, Opt. Spektrosk., 1963, 15, 61.

17 T. H. Huang, K. E. Rieckhoff and E. M. Voigt, J. Chem. Phys., $1982,77,3424$

18 L. Allen and G. I. Peters, Phys. Rev. A, 1973, 8, 2031. 
19 I. Deperasinska, B. Kozankiewicz, I. Biktchantaev and J. Sepioł, J. Phys. Chem. A, 2001, 105, 810.

20 H. Du, R. A. Fuh, J. Li, A. Corkan and J. S. Lindsey, Photochem. Photobiol., 1998, 68, 141.

21 D. R. Tackley, G. Dent and W. E. Smith, Phys. Chem. Chem. Phys., 2001, 3, 1419.
22 V. A. Alekseev, Opt. Spectrosc., 2002, 93, 334; V. A. Alekseev, T. Ridley, K. P. Lawley and R. J. Donovan, Chem. Phys. Lett., 2007, 443, 34; also J. W. Glessner and S. J. Davis, J. Appl. Phys., 1993, 73, 2672.

23 J. Hala, I. Pelant, L. Parma and K. Vacek, Czech. J. Phys. B, 1982, 32, 705 .

\section{STOP!}

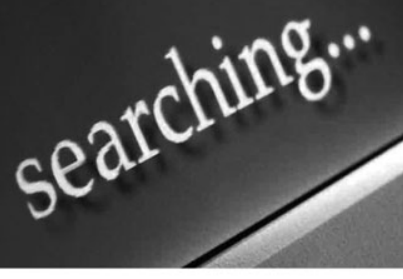

Save valuable time searching for that elusive piece of vital chemical information.

Let us do it for you at the Library and Information Centre of the RSC.

We are your chemical information support, providing:

- Chemical enquiry helpdesk

- Remote access chemical information resources

- Speedy response

- Expert chemical

information specialist staff

Tap into the foremost source of chemical knowledge in Europe and send your enquiries to

\section{library@rsc.org}

